Rapid Reviews COVID-19•

\title{
Review 1: "Non-invasive Vagus Nerve Stimulation for Respiratory Symptoms of COVID-19: Results From a Randomized Controlled Trial (SAVIOR I)"
}

Abrahão Fontes Baptista ${ }^{1}$

${ }^{1}$ Universidade Federal do ABC

Published on: Oct 28, 2021

License: Creative Commons Attribution 4.0 International License (CC-BY 4.0). 


\section{$\underline{\text { RR:C19 Evidence Scale rating by reviewer: }}$}

- Potentially informative. The main claims made are not strongly justified by the methods and data, but may yield some insight. The results and conclusions of the study may resemble those from the hypothetical ideal study, but there is substantial room for doubt. Decision-makers should consider this evidence only with a thorough understanding of its weaknesses, alongside other evidence and theory. Decision-makers should not consider this actionable, unless the weaknesses are clearly understood and there is other theory and evidence to further support it.

$* * * * * * * * * * * * * * * * * * * * * * * * * * * * * * * * * * * * * * * * * *$

\section{Review:}

The manuscript, "Non-invasive Vagus Nerve Stimulation for Respiratory Symptoms of COVID-19: Results From a Randomized Controlled Trial (SAVIOR I)," reports the results from a randomized controlled clinical trial to investigate the safety, efficacy, and feasibility of using non-invasive vagus nerve stimulation (nVNS) during the treatment of hospitalized patients with COVID-19. The study assessed 97 from 110 enrolled participants. Positive results were seen in some inflammatory biomarkers. To my knowledge, this is one of the more extensive studies in the field. It was adequately registered at ClinicalTrials.gov, but its primary objective (reinforced by the title) was to assess the clinical effects of nVNS. However, the results highlight surrogate (inflammatory) and not clinical results. Since there are potential conflicts of interest regarding the study results, using a clinically oriented title and presenting a positive surrogate result may be an essential bias. Also, the authors must state what type of hypothesis they had regarding the results of the study. Were they testing equivalence, non-inferiority, or superiority?

Non-invasive vagus nerve stimulation may be applied with a diversity of devices. For example, adding measures of the Number Needed to Treat or to Harm and costeffectiveness measures would be desired in this study since the actual study used a costly intervention (nVNS with gammaCore Sapphire ${ }^{\mathrm{TM}}$ ).

Other aspects of the study that should be reviewed:

* Statistical differences between study groups at baseline should be presented in table 3;

* CRP is a numerical endpoint with absolute values. Why was this variable analyzed as 
percentages of subjects with normal values? This procedure reduces the possibilities of robust statistical analysis done with numerical values (nominal scale);

* Regarding safety, how was it assessed systematically? Please, provide data regarding instruments used to evaluate the safety, and the participants' responses. 